\title{
INTERFERENCE SELF-MITIGATING BEAMFORMING FOR THE $K$-USER MIMO IC
}

\author{
Jianqi Wang, Amitav Mukherjee and A. Lee Swindlehurst \\ Dept. of Electrical Engineering \& Computer Science \\ University of California Irvine \\ $\{a \cdot m u k h e r j e e ;$ swindle\}@uci.edu
}

\begin{abstract}
This work studies distributed linear transmission strategies for the multiple-input multiple-output (MIMO) interference channel with multiple concurrent links. We introduce the notion of interference self-mitigating beamforming (ISM-BF), where each transmitter minimizes the interference it causes to other users while satisfying its own signal-to-interferenceplus-noise ratio requirement. The computation of the optimal transmit beamformers is shown to decouple into a generalized eigenvector problem. In addition, we analyze the optimality and uniqueness of the proposed distributed beamforming solution. Numerical simulations of the sum rate performance of ISM-BF show it to be a viable alternative to interference alignment-based techniques.
\end{abstract}

\section{INTRODUCTION}

In recent years, significant attention has been devoted to the MIMO interference channel, which is comprised of multiple multi-antenna transmitter-receiver pairs that wish to communicate simultaneously. The concurrent and uncoordinated operation of multiple links naturally gives rise to the presence of interference at each of the receiving nodes. To reduce complexity, the multi-user interference is usually treated as additive noise by the receivers, i.e., multiuser detection is not employed. With this assumption, the resulting tradeoff between maximizing the individual or collective information rates while suppressing interference to reasonable levels has led to a number of transmission strategies being recently proposed.

While the capacity region of interference channels remains unknown in general, transmission strategies that attempt to maximize the sum rate have been studied extensively [1]. A centralized gradient search algorithm to optimize the transmit covariances was introduced in [2]. The lack of cooperation among transmitters in the interference channel leads to a game-theoretic formulation in [3], where each user tries to selfishly maximize their own mutual information. A notable advance was made in $[4,5]$, which introduced the precoding technique of interference alignment (IA) and showed that it could achieve the theoretical multiplexing gain of the MIMO interference channel at high SNR. In [6], two modified versions of IA and a gradient-based centralized algorithm were presented that demonstrated an improvement in sum rate.

In this work, we investigate distributed beamforming strategies for the MIMO interference channel from a different perspective. Specifically, we set each user's objective as the minimization of the sum interference caused to other receivers conditioned upon satisfying their own desired performance criterion. In the context of MIMO broadcast channels, the sum interference caused by a particular user's signal to the other receivers was referred to as 'leakage' in $[7,8]$, where the authors proposed maximizing the downlink signal-to-leakage-plus-noise ratio (SLNR) instead of the traditional signal-to-interference-plus-noise ratio (SINR) of each user in a point-to-point link. However, in the next section we highlight a drawback of this approach and enunciate our alternative design philosophy in the context of the MIMO interference channel.

\section{SYSTEM MODEL}

\subsection{Interference Channel}

We consider an interference channel with $K$ pairs of users, with no coordination allowed between pairs. Each pair is comprised of one transmitter and its intended receiver equipped with $M_{t}$ and $M_{r}$ antennas, respectively. Let $\boldsymbol{H}_{j, i}$ denote the MIMO channel from the $i$ th transmitter to the $j$ th receiver, $\boldsymbol{H}_{j, i} \in \mathbb{C}^{M_{r} \times M_{t}} \quad \forall i, j$. We consider a scalar beamforming strategy where transmitter $k$ 's signal can be written as $\mathbf{x}_{k}=\sqrt{P}_{k} \boldsymbol{v}_{k} s_{k}$, with beamformer $\boldsymbol{v}_{k} \in \mathbb{C}^{M_{t} \times 1}$, unitenergy information symbol $s_{k}$, and transmit power constraint $P_{k}$. Therefore, we impose the restriction $\boldsymbol{v}_{k}^{H} \boldsymbol{v}_{k} \leq 1$.

The received signal at receiver $k$ is

$$
\boldsymbol{y}_{k}=\sqrt{P_{k}} \boldsymbol{H}_{k, k} \boldsymbol{v}_{k} s_{k}+\sum_{j=1, j \neq k}^{K} \sqrt{P_{i}} \boldsymbol{H}_{k, i} \boldsymbol{v}_{i} s_{i}+\boldsymbol{n}_{k},
$$

where $\boldsymbol{n}_{k} \sim \mathcal{C N}\left(\mathbf{0}, \sigma_{k}^{2} \boldsymbol{I}\right)$ denotes complex additive white Gaussian noise. Let the net interference-plus-noise vector be denoted by $\tilde{\boldsymbol{n}}_{k}=\sum_{i=1, i \neq k}^{K} \sqrt{P_{i}} \boldsymbol{H}_{k, i} \boldsymbol{v}_{i} s_{i}+\boldsymbol{n}_{k}$. We there- 
fore have $\tilde{\boldsymbol{n}}_{k} \sim \mathcal{C N}\left(\mathbf{0}, \boldsymbol{F}_{k}\right)$, where

$$
\boldsymbol{F}_{k}=\sum_{i=1, i \neq k}^{K} P_{i} \boldsymbol{H}_{k, i} \boldsymbol{v}_{i} \boldsymbol{v}_{i}^{H} \boldsymbol{H}_{k, i}+\sigma_{k}^{2} \boldsymbol{I} .
$$

Assuming receiver $k$ employs a max-SINR receive beamformer $\mathbf{w}_{k}=\boldsymbol{F}_{k}^{-1} \boldsymbol{H}_{k, k} \boldsymbol{v}_{k}$, the received SINR is

$$
\mathrm{SINR}_{k}=P_{k} \boldsymbol{v}_{k}^{H} \boldsymbol{H}_{k, k}^{H} \boldsymbol{F}_{k}^{-1} \boldsymbol{H}_{k, k} \boldsymbol{v}_{k} .
$$

\subsection{Max-SLNR and Altruistic Beamforming}

In $[7,8]$, the downlink beamforming vectors for the MIMO broadcast channel are chosen so as to maximize the SLNR of each user. The objective is to control the interference (leakage) caused by any user's signal at the unintended receivers. This approach can easily be extended to the MIMO interference channel with distributed transmitters. Specifically, let $\boldsymbol{v}_{1}$ be the beamforming vector for transmitter 1 , for example. The SLNR-maximizing transmit beamformer is obtained as

$$
\hat{\boldsymbol{v}}_{1}=\arg \max _{\left|\boldsymbol{v}_{1}\right|=1} \frac{P_{1} \boldsymbol{v}_{1}^{H} \boldsymbol{H}_{1,1}^{H} \boldsymbol{H}_{1,1} \boldsymbol{v}_{1}}{\boldsymbol{v}_{1}^{H}\left(\sum_{j=2}^{K} P_{1} \boldsymbol{H}_{j, 1}^{H} \boldsymbol{H}_{j, 1}+\sigma_{n}^{2} \mathbf{I}\right) \boldsymbol{v}_{1}},
$$

where the denominator term $\boldsymbol{v}_{1}^{H}\left(\sum_{j=2}^{K} P_{1} \boldsymbol{H}_{j, 1}^{H} \boldsymbol{H}_{j, 1}\right) \boldsymbol{v}_{1}$ denotes the sum interference, i.e., leakage, caused by user 1 .

The drawback of this approach is that it does not guarantee a large effective signal power $\boldsymbol{v}_{1}^{H} \boldsymbol{H}_{1,1}^{H} \boldsymbol{H}_{1,1} \boldsymbol{v}_{1}$. With nonzero probability, the solution to (3) may result in both the signal power and the leakage power being low even though the SLNR is large. In [9], an unconstrained minimization of the sum interference is used to construct the transmit beamformers (defined as the altruistic beamforming method) in a gametheoretic framework. Similar to the preceding discussion, the purely altruistic approach cannot guarantee high SINR, and is known to be sub-optimal in terms of the interference channel sum rate. Since it is desirable to maximize each receiver's receive SINR, such outcomes must be avoided. To this end, the notion of interference self-mitigating beamforming (ISM-BF) is introduced in the next section.

\section{INTERFERENCE SELF-MITIGATING BEAMFORMING}

In this section we present the design of the beamforming vector $\boldsymbol{v}_{1}$ for transmitter 1 , with the same design principle holding true for all other transmitters. Let $\boldsymbol{Q}=\boldsymbol{H}_{1,1}^{H} \boldsymbol{F}_{1}^{-1} \boldsymbol{H}_{1,1}$. The normalized interference caused by transmitter 1 is written as

$$
f\left(\boldsymbol{v}_{1}\right)=\sum_{j=2}^{K} \boldsymbol{v}_{1}^{H} \boldsymbol{H}_{j, 1}^{H} \boldsymbol{H}_{j, 1} \boldsymbol{v}_{1} .
$$

We assume transmitter 1 has knowledge of its outgoing channels $\boldsymbol{H}_{j, i}, j=2, \ldots, K$, and is able to obtain an accurate estimate of the interference-plus-noise covariance $\boldsymbol{F}_{1}$ at receiver 1 . The optimization problem we are seeking to solve is then given by

$$
\boldsymbol{v}_{1}^{*}=\arg \min _{\boldsymbol{v}_{1}} f\left(\boldsymbol{v}_{1}\right)
$$

subject to

$$
\begin{gathered}
\boldsymbol{v}_{1}^{H} \boldsymbol{Q} \boldsymbol{v}_{1} \geq \rho \\
\boldsymbol{v}_{1}^{H} \boldsymbol{v}_{1} \leq 1
\end{gathered}
$$

where the first inequality constraint ensures that the effective (normalized by $P$ ) SINR of receiver 1 is above a predefined threshold $\rho$, and the second inequality constraint corresponds to the normalized transmit power budget. Note that if the largest eigenvalue of $Q$ is smaller than $\rho$, there are no beamforming vectors that satisfy the constraints and the problem is infeasible. Otherwise, the minimizer exists as summarized in the following lemma.

Lemma 1 Let $\mathcal{S}=\left\{\boldsymbol{v}_{1} \mid \boldsymbol{v}_{1}^{H} \boldsymbol{Q} \boldsymbol{v}_{1} \geq \rho, \boldsymbol{v}_{1}^{H} \boldsymbol{v}_{1} \leq 1\right\}$. Let $d_{\max }$ be the largest eigenvalue of $\boldsymbol{Q}$. If $d_{\max } \geq \rho$, there exists $\boldsymbol{v}_{1}^{*}$ such that for any $\boldsymbol{v}_{1} \in \mathcal{S}$, we have $f\left(\boldsymbol{v}_{1}^{*}\right) \leq f\left(\boldsymbol{v}_{1}\right)$.

Proof: The objective function $f(\boldsymbol{v})$ is continuous by definition. When $d_{\max } \geq \rho$, set $\mathcal{S}$ is nonempty. In addition, $\mathcal{S}$ is closed and is contained in the unit ball; therefore it is a compact set. According to the Weierstrass Theorem [10, Thm. 4.2], $f(\boldsymbol{v})$ achieves its minimum on $\mathcal{S}$.

The Lagrangian function for the optimization problem is

$l(\lambda, \mu)=\sum_{j=2}^{K} \boldsymbol{v}_{1}^{H} \boldsymbol{H}_{j, 1}^{H} \boldsymbol{H}_{j, 1} \boldsymbol{v}_{1}+\lambda\left(\rho-\boldsymbol{v}_{1}^{H} \boldsymbol{Q} \boldsymbol{v}_{1}\right)+\mu\left(\boldsymbol{v}_{1}^{H} \boldsymbol{v}_{1}-1\right)$

where $\lambda, \mu \geq 0$. Differentiating (7), we have

$$
\frac{\partial l}{\partial \boldsymbol{v}_{1}}=2 \boldsymbol{P} \boldsymbol{v}_{1}-2 \lambda \boldsymbol{Q} \boldsymbol{v}_{1}+2 \mu \boldsymbol{v}_{1}
$$

where $\boldsymbol{P}=\sum_{j=2}^{N} \boldsymbol{H}_{j, 1}^{H} \boldsymbol{H}_{j, 1}$. The resulting Karush-KuhnTucker (KKT) necessary conditions are

$$
\begin{aligned}
(\boldsymbol{P}+\mu \boldsymbol{I}) \boldsymbol{v}_{1} & =\lambda \boldsymbol{Q} \boldsymbol{v}_{1} \\
\boldsymbol{v}_{1}^{H} \boldsymbol{Q} \boldsymbol{v}_{1} & =\rho \\
\mu\left(\boldsymbol{v}_{1}^{H} \boldsymbol{v}_{1}-1\right) & =0 \\
\boldsymbol{v}_{1}^{H} \boldsymbol{v}_{1}-1 & \leq 0 \\
\lambda>0, \mu & \geq 0 .
\end{aligned}
$$

Let $\lambda_{1} \leq \lambda_{2} \leq \ldots \leq \lambda_{M}$ be the generalized eigenvalues of the matrix pencil $(\boldsymbol{P}+\mu \boldsymbol{I}, \boldsymbol{Q})$, and $\boldsymbol{g}_{1}, \boldsymbol{g}_{2}, \ldots, \boldsymbol{g}_{M}$ be the corresponding generalized eigenvectors with unit norm, i.e., $\left\|\boldsymbol{g}_{i}\right\|=1$, for $i=1, \ldots, M$. If $\boldsymbol{g}_{1}^{H} \boldsymbol{Q g}_{1} \geq \rho$, then $\mu=0$ and the optimal solution is

$$
\boldsymbol{v}_{1}^{*}=\sqrt{\frac{\rho}{\boldsymbol{g}_{1}^{H} \boldsymbol{Q} \boldsymbol{g}_{1}}} \boldsymbol{g}_{1} .
$$


This case corresponds to the one studied in [7]; when $\mu=0$, (9) is the generalized eigenvalue problem considered there.

Lemma 1 gives the conditions under which a solution is guaranteed to exist; the results listed below guarantee the uniqueness of the solution.

Lemma 2 Assume $\boldsymbol{v}_{1}^{*}$ is the solution to the optimization problem (5) with auxiliary parameters $\lambda^{*}$ and $\mu^{*}$. Then $\lambda^{*}$ and $\mu^{*}$ must satisfy

$$
\lambda^{*}=\min _{\boldsymbol{v}_{1} \neq 0} \frac{\boldsymbol{v}_{1}^{H}\left(\boldsymbol{P}+\mu^{*} \boldsymbol{I}\right) \boldsymbol{v}_{1}}{\boldsymbol{v}_{1}^{H} \boldsymbol{Q} \boldsymbol{v}_{1}} .
$$

In other words, $\lambda^{*}$ must be the smallest generalized eigenvalue of the matrix pencil $\left(\boldsymbol{P}+\mu^{*} \boldsymbol{I}, \boldsymbol{Q}\right)$, and $\boldsymbol{v}_{1}^{*}$ is the generalized eigenvector that corresponds to $\lambda^{*}$ with $\left\|\boldsymbol{v}_{1}^{*}\right\|=1$.

Lemma 3 Consider the generalized eigenvalue problem

$$
(\boldsymbol{P}+\mu \boldsymbol{I}) \boldsymbol{g}_{1}=\lambda \boldsymbol{Q} \boldsymbol{g}_{1}
$$

Let $\boldsymbol{g}_{1}$ and $\boldsymbol{g}_{2}$ be the generalized eigenvectors that correspond to the smallest generalized eigenvalues of the matrix pencils $\left(\boldsymbol{P}+\mu_{1} \boldsymbol{I}, \boldsymbol{Q}\right)$ and $\left(\boldsymbol{P}+\mu_{2} \boldsymbol{I}, \boldsymbol{Q}\right)$, with $\boldsymbol{g}_{1}^{H} \boldsymbol{g}_{1}=\boldsymbol{g}_{2}^{H} \boldsymbol{g}_{2}=1$. Then for $\mu_{1} \leq \mu_{2}$, we have

$$
\boldsymbol{g}_{1}{ }^{H} \boldsymbol{Q} \boldsymbol{g}_{1} \leq \boldsymbol{g}_{2}{ }^{H} \boldsymbol{Q} \boldsymbol{g}_{2} .
$$

Theorem 1 The solution to the optimization problem (5) is unique.

Proof: As stated previously, for the case where $\boldsymbol{v}_{1}^{H} \boldsymbol{Q} \boldsymbol{v}_{1} \geq$ $\rho$, where $\boldsymbol{v}_{1}$ is the smallest eigenvector of the matrix pencil $(\boldsymbol{P}, \boldsymbol{Q}), \boldsymbol{v}_{1}^{*}$ in (14) is the solution.

For other cases, we have $\mu \neq 0$ and $\boldsymbol{v}_{1}^{H} \boldsymbol{Q} \boldsymbol{v}_{1} \leq \rho$, where $\boldsymbol{v}_{1}$ is the smallest eigenvector of the matrix pencil $(\boldsymbol{P}, \boldsymbol{Q})$. The KKT conditions are the necessary conditions for the existence of the solution. We can monotonically increase $\mu$ and solve the generalized eigenvalue problem of (9). According to Lemma 3, $\boldsymbol{v}_{1}{ }^{H} \boldsymbol{Q} \boldsymbol{v}_{1}$ is monotonically increasing, where, with a slight abuse of notation, $\boldsymbol{v}_{1}$ is the smallest eigenvector of the matrix pencil $(\boldsymbol{P}+\mu \boldsymbol{I}, \boldsymbol{Q})$. Therefore, when $\boldsymbol{v}_{1}{ }^{H} \boldsymbol{Q} \boldsymbol{v}_{1}$ attains the value of $\rho$, we obtain the solution and it is unique.

The procedure for finding the unique ISM-BF solution is outlined as follows:

1. Set $\mu=0$, solve the generalized eigenvalue problem

$$
\boldsymbol{P} \boldsymbol{x}=\lambda \boldsymbol{Q} \boldsymbol{x},
$$

and obtain the eigenvector $\boldsymbol{g}_{1}$ that corresponds to the smallest eigenvalue.

2. If $\boldsymbol{g}_{1}^{H} \boldsymbol{Q g}_{1} \geq \rho$, we have the solution for $\boldsymbol{v}_{1}^{*}$ given in (14). Otherwise, perform a line search for $\mu$ that results in $\boldsymbol{v}_{1}^{H} \boldsymbol{Q} \boldsymbol{v}_{1}=\rho$.

\section{DISTRIBUTED IMPLEMENTATION}

We adopt a game-theoretic approach to investigate the convergence and distributed implementation of ISM-BF. Consider the strategic, non-cooperative game $\Omega=\left\{\mathcal{K}, \mathcal{V},\left\{\varphi_{k}\right\}_{k \in \mathcal{K}}\right\}$, where $\mathcal{K}(|\mathcal{K}|=K)$ is the set of all users, each user has a strategy space $\mathcal{V}_{k}, \mathcal{V} \subseteq \mathbb{C}^{K M_{t}}$ is the compact global strategy set of user transmit beamforming vectors, and $\varphi_{k}=-f\left(\boldsymbol{v}_{k}\right)$ is the constrained payoff function for user $k$, with $f\left(\boldsymbol{v}_{k}\right)$ defined as in (4) and the accompanying constraints in (6). The beamforming choices of the competing users affect user $k$ 's payoff and best response implicitly via these constraints.

Next, we recast $\Omega$ as a coordination game with a common objective function $P(\boldsymbol{V}), \boldsymbol{V}=\left[\boldsymbol{v}_{1}, \ldots, \boldsymbol{v}_{K}\right] \in \mathcal{V}$ :

$$
P(\boldsymbol{V})=-\sum_{k=1}^{K} f\left(\boldsymbol{v}_{k}\right)=-\sum_{k=1}^{K} \sum_{j \neq k}^{K} \boldsymbol{v}_{k}^{H} \mathbf{H}_{j, k}^{H} \mathbf{H}_{j, k} \boldsymbol{v}_{k} .
$$

Let $\boldsymbol{v}_{k}, \boldsymbol{v}_{k}^{\prime} \in \mathcal{V}_{k}$ be two valid strategy choices for user $k$. It is easy to show the following:

$\varphi_{k}\left(\boldsymbol{v}_{k} \mid \boldsymbol{V}_{-k}\right)-\varphi_{k}\left(\boldsymbol{v}_{k}^{\prime} \mid \boldsymbol{V}_{-k}\right)=P\left(\boldsymbol{v}_{k} \mid \boldsymbol{V}_{-k}\right)-P\left(\boldsymbol{v}_{k}^{\prime} \mid \boldsymbol{V}_{-k}\right)$

where $\boldsymbol{V}_{-k}$ is the strategy choice of all players except the $k^{\text {th }}$ user, obtained by removing the $k^{t h}$ column of $\boldsymbol{V}$. Now, (17) indicates that the function $P(\boldsymbol{V})$ satisfies the definition of a potential function, and $\Omega$ is an exact potential game [11]. Furthermore, $f\left(\boldsymbol{v}_{k}\right)$ is quadratic (and convex) in $\boldsymbol{v}_{k}$ and in addition $\boldsymbol{H}_{j, k}^{H} \boldsymbol{H}_{j, k}$ is Hermitian positive definite [c.f. (4)], implying $\varphi_{k}$ is strictly concave in $\boldsymbol{v}_{k}$. Potential $P(\boldsymbol{V})$ is a sum of concave functions and is also concave. Therefore, by [11, Theorems 2,3] the potential game $\Omega$ admits at least one Nash Equilibrium since the set of maxima on $P(\boldsymbol{V})$ is nonempty.

In order to reach an equilibrium in a distributed manner, the game is played over multiple stages in an iterative manner. In each stage, the users update their beamforming strategies sequentially (e.g., via the Gauss-Seidel algorithm) using a best-response decision rule [11], which is provably convergent. Numerically, we observed that even if a particular user's SINR target is not achieved, the eventual convergence of the network is not disrupted (convergence in this case is considered to be achieved when a NE of the game is reached).

\section{NUMERICAL RESULTS}

In this section, we present simulation results for several network scenarios, where results for each scenario are obtained by averaging over 1000 Monte Carlo trials. We set the number of users as $K=3$ with $M=4$ antennas to facilitate comparisons with the IA schemes of [4]-[6]. The SNR is defined as the transmit power $P$ since we set $\sigma_{k}^{2}=1 \forall k$. At the first iteration, ISM-BF is initialized with random beamformers.

In Fig. 1, the performance of the ISM-BF technique is compared with the distributed IA approach presented in [5]. 


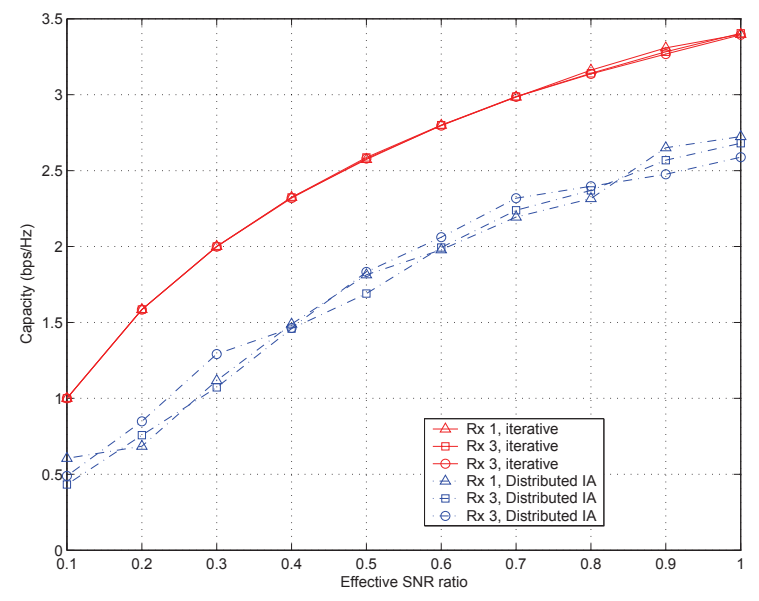

Fig. 1. User rates of ISM-BF and distributed IA.

We can see that our approach performs better for the collective as well as individual rates, while the experimental rate of convergence is of the same order. This occurs even though the ISM-BF algorithm does not directly maximize the network sum rate.

In Fig. 2, we compare the iterative ISM-BF method with the centralized MMSE-IA and gradient search algorithms [6]. We observe that the ISM-BF method offers a performance advantage in the low to intermediate SNR range where interference is dominant, but does not attain the high-SNR multiplexing gain of the centralized schemes.

\section{CONCLUSIONS}

In this work we introduced the concept of interference selfmitigating beamforming (ISM-BF), where each transmitter

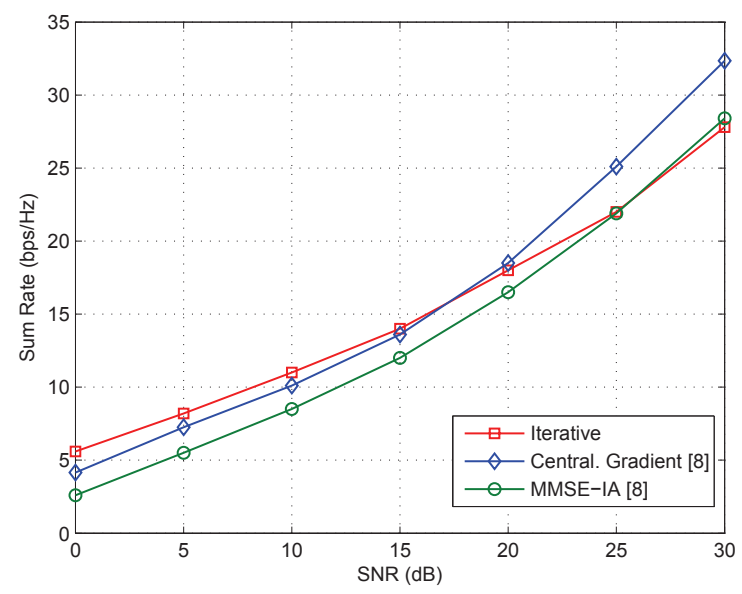

Fig. 2. Sum rate of ISM-BF, centralized MMSE-IA, and gradient search versus SNR. minimizes the interference it causes to other users while satisfying its own SINR requirement. The computation of the optimal transmit beamformers is shown to decouple into a generalized eigenvector problem. In addition, we analyze the optimality and convergence of the proposed distributed beamforming solution. Numerical simulations verify the sum rate performance of ISM-BF compared to interference alignmentbased techniques for a number of network scenarios.

\section{REFERENCES}

[1] X. Shang, B. Chen, and M. J. Gans, "On the achievable sum rate for MIMO interference channels," IEEE Trans. Inf. Theory, vol. 52, pp. 4313-4320, Sep. 2006.

[2] S. Ye and R. S. Blum, "Optimized signaling for MIMO interference systems with feedback," IEEE Trans. Sig. Proc., vol. 51, pp. 2839-2848, Nov. 2003.

[3] G. Scutari, D. P. Palomar, and S. Barbarossa, "Competitive design of multiuser MIMO systems based on game theory: A unified view," IEEE JSAC, vol. 25, pp. 10891103, Sep. 2008.

[4] V. R. Cadambe and S. A. Jafar, "Interference alignment and the degrees of freedom for the $K$-user interference channel, IEEE Trans. Inf. Theory, vol. 54, pp. 3425-3441, Aug. 2008.

[5] K. S. Gomadam, V. R. Cadambe, and S. A. Jafar, "Approaching the capacity of wireless networks through distributed interference alignment," [Online]. Available at: http://arxiv.org/abs/0803.3816

[6] H. Sung, S.-H. Park, K.-J. Lee, and I. Lee, "Linear precoder designs for $K$-user interference channels", IEEE Trans. Wireless Commun., vol. 9, pp. 291-301, Jan. 2010.

[7] M. Sadek, A. Tarighat, and A. H. Sayed, "Active antenna selection in multiuser MIMO communications," IEEE Trans. Sig. Proc., vol. 55, pp. 1498-1510, Apr. 2007.

[8] M. Sadek, A. Tarighat, and A. H. Sayed, "A leakagebased precoding scheme for downlink multi-user MIMO channels," IEEE Trans. Wireless Commun., vol. 6, pp. 1711-1721, May 2007.

[9] Z. M. Ho and D. Gesbert, "Balancing egoism and altruism on the MIMO interference channel," Proc. ICC, 2010.

[10] E. K. P. Chong and S. H. Zak, An Introduction To Optimization. John Wiley, 2001.

[11] G. Scutari, S. Barbarossa, and D. P. Palomar, "Potential games: A framework for vector power control problems with coupled constraints," Proc. ICASSP, 2006. 